\title{
Cloud Assisted Mobile Multimedia Streaming -A Network and Device Aware Novel DASRC Prediction Scheme
}

\author{
Abhinaya.A \\ IInd Year - M.E. CSE \\ Srinivasan Engineering College \\ Perambalur
}

\author{
Karthikeyan.B \\ Professor / ECE \\ Srinivasan Engineering College \\ Perambalur
}

\begin{abstract}
Mobile streaming with the use of mobile devices nowadays is widespread and multimedia provides the best way to understand, learn new concepts and technologies in the time of their birth. Mobile learning will provide more realistic approach as it will keep everyone with just in term learning on the move. In general it's not possible for everyone to afford huge investments on hardware and software resources. This is the main reason for stepping over to the cloud where it provides centralized storage, memory, processing and bandwidth which maps the user end terminal via interactive mobile streaming services by adjusting the transmission frequency. This study outlines the state of art and major research challenges in architectures, protocols in streaming which contributes to good scalability with minor delay and soaring playback inconsistencies. Further, the DASRC algorithm considers the data download rate, as well as theenergy for decoding is calculated before the video data is transmitted. DASRC algorithm also evaluates the picture quality, available bandwidth and energy consumption for the user end terminal device. The feasibility of the proposed prototype is validated with architecture to yield high performance for network, device and energy aware QoS approach.
\end{abstract}

\section{Keywords}

Adaptive QoS (Quality of Service), Mobile streaming, Cloud multimedia, Network and Device aware, DASRC (Dynamic Adaptive Spatial Resolution Control).

\section{INTRODUCTION}

The rise of the cloud is more than just another platform shift that gets everyone excited. It will allow digital technology to penetrate every nook and corner of the economy and of society. Cloud has already proliferated various facets of our daily life. We can see its applications in education, communications, financial services, healthcare, energy, entertainment, collaborations utility industries, and many more. Cloud definitely can manage multimedia contents.

Multimedia content dissemination is a complex and challenging task. Even though the general-purpose cloud computing system is good for most of the data services, it is been not quite adequate to meet the stringent quality of service or QoS (bandwidth, latency, and jitter), and quality of experience or QoE for multimedia applications. If it's not properly designed, then cloud can suffer from a degraded QoS or QoE, although it can provide the processing power, storage, multimedia services hosted on web. Mobile devices have limited capacity in terms of storage, memory and processing power. As of now, delivery of multimedia content to mobile devices through cloud computing demands even more stringent QoS provisioning than the fixed network devices. The best practice is to offload storage and computing to cloud without downgrading mutual interactivity, and quantify steps to prevent any sort of latency.

\section{RELATED WORK}

Multimedia cloud computing can be classified into two major divisions. One is media aware cloud, and the other is cloudaware multimedia studies related to this study as follows.

\subsection{Analysis of Multimedia-Aware Cloud}

Techniques for configuring and operating a multimedia aware cloud are particularly configured for mobile device computing. Clusters of servers are organized for general computing and data storage. A load balancing server can be configured to: identify multimedia file formats currently being processed within the multimedia edge cloud; determine desired QoS levels for each multimedia format identified; evaluate individual device communicating abilities with the multimedia edge cloud; and assess bandwidth of each network over which the multimedia edge cloud with which mobile device communicates. With this, multimedia data may be adapted accordingly, to result in an acceptable QoS level when delivered to a specific mobile device. The multimediaaware cloud mainly aims on the media files in the cloud environment. Meeting the requirement of QoS, according to the arithmetic capability of the cloud [1], [2], involves the balanced sharing of the load, real-time dynamic coding and heterogeneous work conversions. Fan et al. [3] used job scheduling for various clusters in the cloud environment to attain load balance. Zheng et al. [4] proposed a collaborative quality ranking framework and used the greedy mode to calculate the speeds of various components in the cloud, in order to efficiently improve the overall applications. DiazSanchez et al. [5] used the Digital Living Network Alliance (DLNA) and Universal Plug and Play (UPnP) as an open multimedia platform for domestic multimedia sharing, and then used either the http or UDP transmission protocol to obtain open cloud computing middleware. Fengy et al. [6] proposed streaming-oriented management service architecture according to different multimedia data in the cloud environment.

\subsection{Analysis of Cloud-Aware Multimedia}

Multimedia cloud is very similar in concept to content delivery network (CDN) which involves delivering multimedia content from the edge locations rather than original servers. In such scenario computation is performed at edge locations rather than one central cloud in order to reduce latency and computation time and improve response time. Differing from the multimedia-aware cloud, cloud-aware multimedia inclines to the streaming service of front-end 
multimedia data. Multimedia video files are usually quite large. In order to provide the multimedia streaming service of real-time videos, files are divided into packets, so that the client-side can view multimedia information instantly as received instead through downloading. Multimedia streaming usually contains several major elements, such as the encoder, the decoder, the streaming server and the player. Streaming technology can be classified into two types: the web server and the streaming server. The web server is a general web server, using Hyper- Text Transfer Protocol (HTTP) as the communication medium. Streaming in this mode is called HTTP streaming. It is convenient because no additional streaming servers are required; therefore it is also known as server less streaming. The HTTP protocol uses Transmission Control Protocol (TCP). A handshake must be done before transmission, and retransmission is required if a data packet is lost, which causes severe delay. Therefore, a set of HTTP live streaming protocols was proposed for Apple Inc., which provided streaming services that allowed packet interruption. The http live streaming transmission mode to transcode multimedia files at the mobile device side for live streaming. An applicable bandwidth is allocated in environment to each mobile device properly in a P2P streaming environment, used game formulation to model the collaborative streaming problem, and predicted the transmission probability of each peer to calculate the overall bandwidth allocation. Trajkovska et al. [7] proposed a $\mathrm{QoS}$ function for the heterogeneous architecture of a cloud environment. They calculated the QoS parameter environment of each client side and executed QoS services according to the hybrid environment. The interactive streaming service aims to provide preferable mutual information between the server and client-side. Liu et al. [8] proposed a recommendation service that transmits recommended multimedia browse file to mobile devices according to the supported resolution fed back from the mobile devices. The present contemporary ubiquitous media services and recommended multimedia files functions to direct resizing, attention-based adaptation, and user guided semantic adaptation models. It considered the arithmetic capability and resolution of the terminal unit environment and the power factor at the mobile device side for the selection of multimedia streaming. Multimedia content providers have started using cloud computing for on-demand video and real streaming. This envisions that with the emergence of open standards in cloud computing and multimedia content, the future will see a seamless multimedia cloud computing experience for users.

\subsection{Analysis of Dynamic SVC Multimedia Service}

SVC contains spatial, temporal or quality three-dimensional parameters that can be adjusted dynamically and are provided in multilayer transmission, so as to provide a preferable streaming service for network instability, which has been mostly studied in mobile multimedia services. The methods proposed by Sohn et al. [9] and Choi et al. [10] assures the spatial, temporal or quality layer number preferentially according to the user's preference setting, and gives the largest number of SVC layers within the capacity of the available bandwidth to match the variance in the network environment and to increase the bandwidth utilization rate. A cloud-based SVC proxy suits for the SVC format conversion and transmission of multimedia files to provide better streaming services. The Priority based Layer Switching (PLS) algorithm determines the receiving bit rate according to RTCP and changes the SVC layer quantity according to the receiving bit rate, in order to match variances in the network environment. The prediction of the available bandwidth according to the history and network monitoring, and then abstracting the image data containing partial SVC layers for transmission, allowing the streaming the bit rate to approach to the predicted available bandwidth is then carried on as usual. Differing from only considering the network quality, the device capability is an important factor that limits video quality. The proposed Device and Network-Aware Scaling (DeNAS) for multiple mobile devices, determines the appropriate SVC layer number according to the device capability, the network capacity and the user's request, so as to meet the video quality requirement of the device and to increase the efficiency of the network bandwidth service. The results showed that even in multiple shared environments with limited bandwidth, the framework can provide SVC streaming with preferable image quality. Lee et al. [11] proposed the Adaptive Spatial Resolution Control (ASRC) algorithm in order to reduce the power consumption of mobile devices. This algorithm considers picture quality, available bandwidth and energy consumption. The energy for decoding is calculated before the video data is transmitted, and the energy consumption and picture quality are evaluated before the SVC layer number to be downloaded is determined.

\section{SYSTEM ARCHITECTURE}

The proposed system provided an efficient interactive streaming service for diversified mobile devices and dynamic network environments. The overall system structure is shown in the below Fig.1 When a mobile device requests a multimedia streaming service, it transmits its hardware and network environment parameters to the profile agent in the cloud environment, which records the mobile device codes and determines the required parameters, and then transmits them to the Network and Device negotiation Control Module (NDNCM).

The NDNCM determines the most suitable SVC code for the device according to the parameters, states, conditions and then the SVC Transcoding Controller (STC) hands over the transcoding work via map-reduce to the cloud, in order to increase the transcoding rate. Finally, the multimedia video file is transmitted to the mobile device through the service.

Seamless streaming of multimedia content ensures Quality of Service (QoS) over heterogeneous networks is a desired feature for several multimedia services, hence the multimedia contents should be adapted to various environment usages such as network and device characteristics, terminal capabilities, and user expectations. Scalability in video coding is a good feature to meet the requirement of heterogeneous networks. 


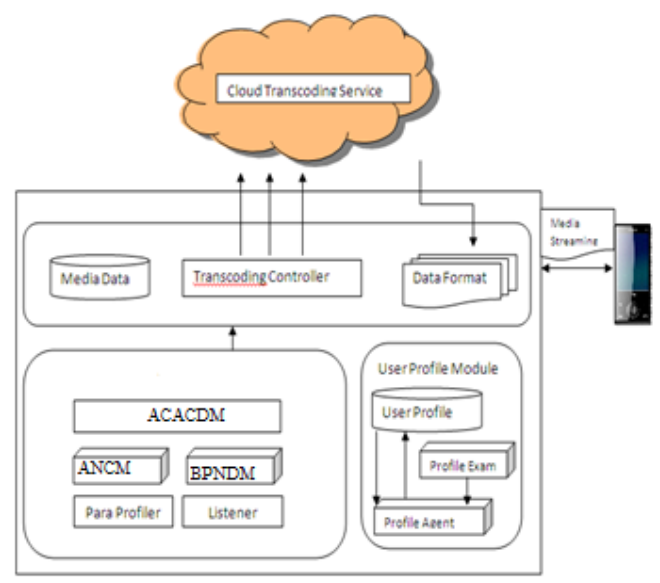

Fig 1.Cloud assisted Mobile Multimedia Streaming

\subsection{Modeling User Profile Activities}

The profile negotiator (agent) is used to receive the mobile hardware environment parameters and create a user profile. The mobile device transmits its hardware specifications in XML-schema format to the profile negotiator in the cloud server, as shown in the following Table I. The XML-schema is metadata, which is mainly semantic and assists in describing the data format of the file.

TABLE I

DATA STRUCTURE OF FEATURE PARAMATERS

\begin{tabular}{|l|l|l|}
\hline Data type & Function & Description \\
\hline Int & Resolution & Supportable resolution \\
\hline Float & CPU & Operation ratio \\
\hline Float & Battery & Dump energy of the device \\
\hline Float & $\mathrm{T}_{\mathrm{vs}}$ & Start time of video decoding \\
\hline Float & $\mathrm{T}_{\mathrm{ve}}$ & End time of video decoding \\
\hline Float & Bit rate & Video bit rate \\
\hline Float & Bandwidth & Tested existing bandwidth \\
\hline
\end{tabular}

TABLE II

DATA STRUCTURE OF PREDICTION PARAMETERS

\begin{tabular}{|l|l|l|}
\hline Data type & Function & Description \\
\hline Int & $\mathrm{R}_{\mathrm{w}}$ & Width of resolution \\
\hline Int & $\mathrm{R}_{\mathrm{h}}$ & Height of resolution \\
\hline Float & $\mathrm{CP}_{\mathrm{avg}}$ & Average value of CPU \\
\hline Float & $\mathrm{CP}$ & Current operation ratio \\
\hline Float & $\mathrm{Db}$ & Dump energy of device \\
\hline Float & $\mathrm{T}_{\mathrm{vs}}$ & Start time of video encoding \\
\hline Float & $\mathrm{T}_{\mathrm{ve}}$ & End time of video encoding \\
\hline Float & $\mathrm{Bits}$ & Video bit rate \\
\hline Float & $\mathrm{Bw}_{\mathrm{w}}$ & Tested existing bandwidth \\
\hline Float & $\mathrm{Bw}_{\mathrm{avg}}$ & Average available bandwidth \\
\hline Float & $\mathrm{Bw}_{\mathrm{std}}$ & $\begin{array}{l}\text { Standard deviation of } \\
\text { bandwidth }\end{array}$ \\
\hline
\end{tabular}

The metadata enables non-owner users to see information about the files, and its structure is extensible. However, any mobile device that is using this cloud service for the first time will be unable to provide such a profile, so there shall be an additional profile examination to provide the test performance of the mobile device and sample relevant information. Through this function, the mobile device can generate an XML-schema profile and transmit it to the profile agent. The profile agent determines the required parameters for the XML-schema and creates a user profile, and then transmits the profile to the NDNCM for identification.

\subsection{Network and Device Negotiation Control Module (NDNCM)}

The NDNCM aims to determine the interactive communication frequency and the SVC multimedia file coding parameters according to the parameters of the mobile device. It hands these over to the STC for transcoding control, so as to reduce the communication bandwidth requirements and meet the mobile device user's demand for multimedia streaming. It consists of a listen module, a parameter profile module, a network estimation module, a device-aware Bayesian prediction module, and adaptive multi-layer selection. The interactive multimedia streaming service must receive the user profile of the mobile device instantly through the listen module. The parameter profile module records the user profile and determines the parameter list required in this study, as shown in Table II. This is provided to both the network estimation module and the device-aware Bayesian prediction module to predict the required numerical values. $R_{w}$ and $R_{h}$ represent the width and height of the supportable resolution for the device, and $\mathrm{CP}_{\mathrm{avg}}$ and $\mathrm{CP}$ represent the present and average $\mathrm{CPU}$ operating speed. $\mathrm{Db}$ and $\mathrm{Db}_{\text {rate }}$ represent the existing energy of the mobile device and energy consumption rate, $\mathrm{Bw}, \mathrm{Bw}_{\mathrm{avg}}$ and $\mathrm{Bw}_{\text {std }}$ represent the existing, average and standard deviation values of the bandwidth. When this parameter form is maintained, the parameters can be transmitted to the network estimation module and the device- aware Bayesian prediction module for relevant prediction.

\subsection{Active Network Computation Module (ANCM)}

The ANCM is mainly based on the measurement-based prediction concept however; it further develops the Exponentially Weighted Moving Average (EWMA). The EWMA uses the weights of the historical data and the current observed value to calculate gentle and flexible network bandwidth data for the dynamic adjustment of weights. In order to determine the precise network bandwidth value, the EWMA filter estimates the network bandwidth value. $\mathrm{Bw}_{\mathrm{est}}{ }^{(t)}$ is the estimated bandwidth of the No. $\mathrm{t}$ time interval, $\mathrm{Bw}^{(\mathrm{t}-1)}$ is the bandwidth of the No. time t-1 interval, $\mathrm{B}_{\text {std }}$ being the standard bandwidth and $\mathrm{D}$ is the estimation difference.

$\mathrm{Bw}_{\mathrm{est}}^{(\mathrm{t})}=\mathrm{Bw}^{(\mathrm{t}-1)}+\mathrm{D}^{(\mathrm{t})}$

The error correction of estimation and the overall standard difference and estimated the different bandwidths is obtained by adjusting the weights:

$\mathrm{D}^{(\mathrm{t})}=\left(1-\alpha^{(\mathrm{t})}-\beta^{(\mathrm{t})}\right) \mathrm{D}^{(\mathrm{t}-1)}+\alpha^{(\mathrm{t})}\left(\mathrm{Bw}^{(\mathrm{t}-1)}-\mathrm{Bw}^{(\mathrm{t}-}\right.$
$\left.{ }^{2)}\right)+\beta(\mathrm{t}) \mathrm{Bw}_{\text {std }}$

In a mobile environment, the modifier formula for the two weights is shown as: 
$\alpha^{(\mathrm{t})}=\alpha^{(\mathrm{t}-1)}(1-\gamma)$

Where $\gamma>0$, if $\left\|\mathrm{Bw}_{\mathrm{est}}{ }^{(\mathrm{t})}-\mathrm{Bw}_{\mathrm{est}}{ }^{(\mathrm{t}-1)}\right\|>\mathrm{e}$

$$
\gamma<0 \text {, if }\left\|\mathrm{Bw}_{\text {est }}{ }^{(\mathrm{t})}-\mathrm{Bw}_{\mathrm{est}}{ }^{(\mathrm{t}-1)}\right\|<\mathrm{e}
$$

And $\quad \beta^{(t)}=\beta^{(t-1)}(1-\xi)$,

Where $\xi>0$, if $\left\|\mathrm{Bw}^{(\mathrm{t}-1)}-\mathrm{Bw}_{\mathrm{avg}}\right\|>\mathrm{Bw}_{\text {std }}$

$$
\xi<0 \text {, if }\left\|\mathrm{Bw}^{(\mathrm{t}-1)}-\mathrm{Bw}_{\mathrm{avg}}\right\|<\mathrm{Bw}_{\text {std }}
$$

Among which, $\gamma$ and $\xi$ are the corrected parameters of the Weights and $\mathrm{e}$ is the error boundary value. If the present mobile network is in a stable state, it shall conform to the following equation:

$$
\begin{aligned}
& \mathrm{Bw}_{\text {avg }}{ }^{(\mathrm{t}-1)}+3\left(\mathrm{Bw}_{\text {std }}{ }^{(\mathrm{t}-1)}\right) / \mathrm{d}>\mathrm{Bw}^{(\mathrm{t})}>\mathrm{Bw}_{\mathrm{avg}}{ }^{(\mathrm{t}-1)}- \\
& 3\left(\mathrm{Bw}_{\text {std }}{ }^{(\mathrm{t}-1)}\right) / \mathrm{d}
\end{aligned}
$$

\subsection{Prefiguring Bayesian Network and Device-Aware Module (PBNDAM)}

The SVC hierarchical structure provides scalability of the temporal, spatial and quality dimensions. It adjusts along with the FPS, resolution and video variations of a streaming bitrates; however, the question remains of how to choose an appropriate video format according to the available resources of various devices. The fig adopts the video and device feature in order to conform to the real-time requirements of mobile multimedia. This study adopted Bayesian theory to infer whether the video features conformed to the decoding action.

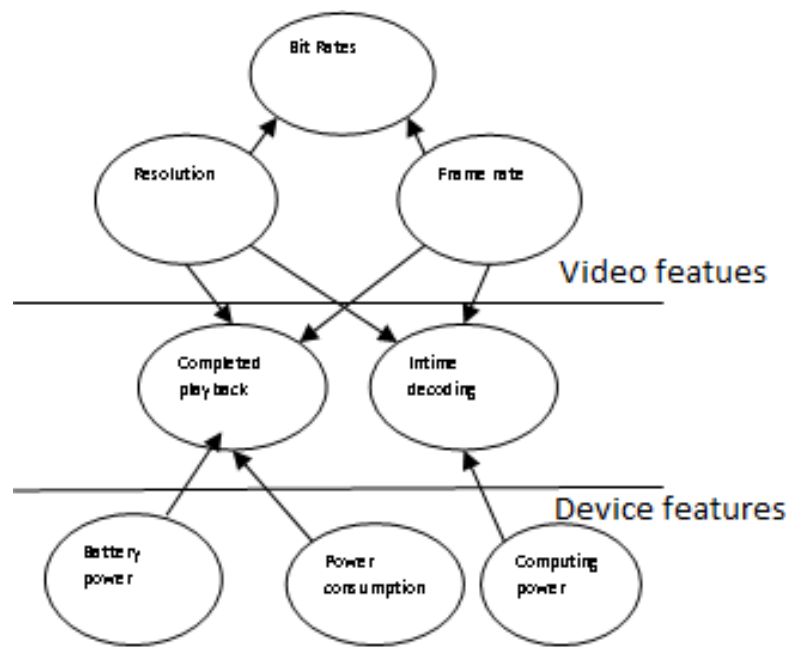

Fig 2.Network and Device parameters

If, $\mathrm{R}=\mathrm{R} 1, \mathrm{R} 2 \ldots \mathrm{RN}, \mathrm{F}=\mathrm{F} 1, \mathrm{~F} 2 \ldots \mathrm{FN}$, in which $\mathrm{R}$ is the aggregate of the resolutions supported by the mobile device, and $\mathrm{F}$ is the aggregate of videos at different frame rates. Event $\zeta$ is set as the event that can be decoded smoothly, and $\eta$ is the event of video completely decoded by the device, defined as:

$$
\zeta: \mathrm{T}_{\mathrm{dec}}<\mathrm{T}_{\mathrm{seg}}
$$

$$
\eta: T_{\text {battery }}<T_{\text {play }}
$$

It is necessary to determine at what resolution and frame rate an entire multimedia file can be decoded completely and smoothly. Considering the below $\mathrm{R}, \mathrm{F}, \zeta, \eta$ and $\mathrm{CP}$ are independent events:

$$
\begin{aligned}
\mathrm{P}\left(\zeta, \eta \mid \mathrm{R}=\mathrm{R}_{\mathrm{i}}, \mathrm{F}=\mathrm{F}_{\mathrm{j}}, \mathrm{CP}\right)=\mathrm{P}\left(\zeta \mid \mathrm{R}=\mathrm{R}_{\mathrm{i}}, \mathrm{F}=\mathrm{F}_{\mathrm{j}}, \mathrm{CP}\right) * \mathrm{P}\left(\eta \mid \mathrm{R}=\mathrm{R}_{\mathrm{i}}\right. \\
\left., \mathrm{F}=\mathrm{F}_{\mathrm{j}}, \mathrm{CP}\right) \\
\mathrm{P}\left(\zeta \mid \mathrm{R}=\mathrm{R}_{\mathrm{i}}, \mathrm{F}=\mathrm{F}_{\mathrm{j}}, \mathrm{CP}\right)=\mathrm{P}\left(\zeta \mid \mathrm{R}=\mathrm{R}_{\mathrm{i}}\right) * \mathrm{P}\left(\zeta \mid \mathrm{F}=\mathrm{F}_{\mathrm{j}}\right) * \mathrm{P}(\zeta \mid \mathrm{CP}) \\
=\left(\mathrm{P}\left(\mathrm{R}=\mathrm{R}_{\mathrm{i}} \mid \zeta\right) * \mathrm{P}(\zeta)\right) / \mathrm{P}\left(\mathrm{R}=\mathrm{R}_{\mathrm{i}}\right) * \\
\left(\mathrm{P}\left(\mathrm{F}=\mathrm{F}_{\mathrm{j}} \mid \zeta\right)^{*} \mathrm{P}(\zeta)\right) / \mathrm{P}\left(\mathrm{F}=\mathrm{F}_{\mathrm{j}}\right) * \\
(\mathrm{P}(\mathrm{CP} \mid \zeta) * \mathrm{P}(\zeta)) / \mathrm{P}(\mathrm{CP})
\end{aligned}
$$

As for the influence of power on video decoding time, according to the power consumption of the processor:

Pow $=\mathrm{C}_{\mathrm{eff}} * \mathrm{~V} 2_{\mathrm{dd}} * \mathrm{~F}$

Among which, $\mathrm{C}_{\text {eff }}$ is the effective switched capacitance, $\mathrm{V}_{\mathrm{dd}}$ represents the operating voltage, and is the operating frequency. The relationship between frequency and voltage is expressed as the following equation:

$\mathrm{F}=\mathrm{K} *\left(\mathrm{~V}_{\mathrm{dd}}-\mathrm{V}_{\mathrm{t}}\right)^{\alpha} / \mathrm{V}_{\mathrm{dd}}$

Among which, $\mathrm{K}$ is a constant, $\mathrm{V}_{\mathrm{t}}$ represents the threshold voltage, the time spent on executing a task is called the workload and is defined as $\mathrm{T}_{\text {proc }}$. $\mathrm{T}_{\text {proc }}$ can be calculated by the following equation:

$\mathrm{T}_{\text {proc }}=\mathrm{C} / \mathrm{F}=1 / \mathrm{F}=1 /$ pow

Among which, represents the cycle number for the workload. Equation (10) indicates that although accelerating the operating frequency can reduce the workload effectively, it relatively accelerates the consumption of power.

\subsection{Flexible Transmission and Multi-layer Content Determination (FTMCD) with DASRC Scheme}

When the predicted bandwidth state and the Bayesian predictive network are determined, the cloud system will further determine the communication and the required multimedia video files according to the information as stated below

\subsubsection{Communication decision}

A good dynamic communication mechanism can reduce the bandwidth needs and the power consumption of the device resulting from excessive packet transmission, and the transmission frequency can be determined according to the bandwidth and its fluctuation ratio based on such dynamic decision-making. The transmit mode is engaged until the device finds a variation of the transmitted variables that exceeds a threshold. Although the threshold can reduce the communication frequency effectively and precisely, in this mode the mobile device must start up additional threads for 
continuous monitoring; thus, the load on the device side is increased.

\subsubsection{SVC multi-layer content selection}

SVC is an improvement over traditional H.264/MPEG-4 AVC coding, as it has higher coding flexibility. It is characterized by the temporal, spatial and SNR scalability, allowing video transmissions to be more adaptable to heterogeneous network bandwidth. When the mobile device transmits the current network and hardware features to the cloud environment, the NDNCM will predict the bandwidth at the next time point according to the bandwidth and standard deviation and will identify whether the bandwidth state is stable or not. The BPNDM infers whether the multimedia video, at different resolutions and frame rates, can complete smooth decoding and whether the hardware can provide complete video playback services, according to the profile examination and subsequent hardware features. When the Bayesian inference table is completed, the next communication time can be determined, and the SVC multimedia coding applicable for the mobile device can be provided according to the predicted and inferred network and hardware features.

\section{PROPOSED WORK}

This research targets the characteristic of streaming protocols to record the current stream video content and the bandwidth state of the user while also analyzing the past bandwidth fluctuations to evaluate and predict the possible bandwidth changes in the future while using map and reduce algorithm in cloud to immediately transfer the video encoding and to quickly transfer the most suitable video format for the user. It considers both power consumption and streaming quality improvement.

Different from mobile streaming, this work introduces cloudbased real time trans-coding for adaptive mobile streaming. Based on cloud computing this researches offers a more efficient method that uses map-reduce to separate the video content into different clips. It also Performs distributed encoding to solve the immediate multimedia trans-coding problem and further introduce a dynamic communicative and predictive bandwidth for dynamic mobile environments. This can provide a more stable streaming service for an unstable network. Compared to a stable network, the losses of bandwidth and power in the terminal units caused by excessive packet transmission can be reduced. The streaming mechanism considers whether the overall electric consumption of the device could provide a complete multimedia file playback service.

The feasibility of the proposed method was validated and the related experiment was analyzed by constructing the prototype environment. The SVC Trans-coding Controller (STC) hands over the trans-coding work via map-reduce to the cloud, in order to increase the trans-coding rate as shown in the figure.

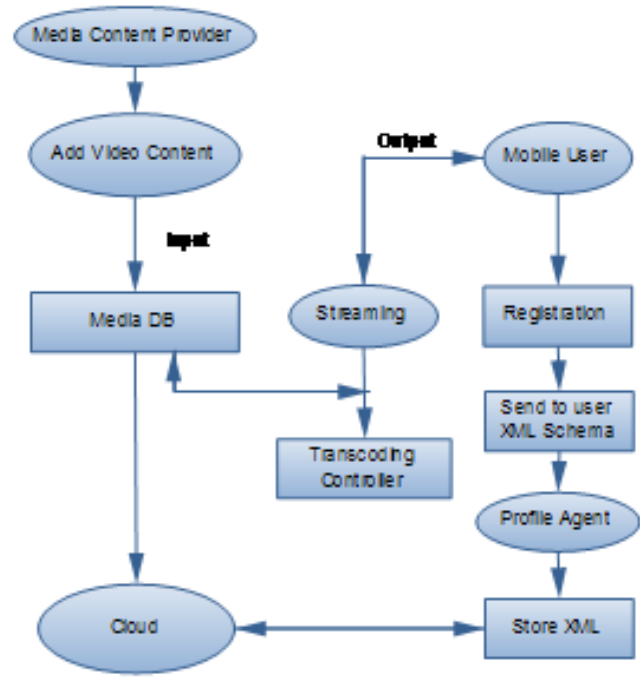

Fig 3.Flow diagram of Mobile Multimedia Streaming

\subsection{Enhancement}

My proposed implementation are designing Cloud based Mobile Streaming to seamlessly utilize an agile resource support and rich functionalities offered by both an IaaS (Infrastructure-as-a-Service) cloud and a PaaS (Platform-asa- Service) cloud. The earlier research works were also based on making the best implementations to get the expected outcome with the cloud assisted mobile media streaming. This research targets and mainly overcomes the issues associated with streaming only for higher bandwidth; quality of encoding and decoding knowledge; appropriate video quality provisioning; inconsistencies an playback service etc.

By adopting the new DASRC scheme, it can be assured that the above stated issues can be overcome to meet increased image resolution, codec knowledge, complete playback service, battery and computational power consumption. DASRC scheme considers the picture quality available bandwidth and energy consumption; it also involves estimation of decoding energy required before actual transmission takes place. Further the number of data's being downloaded in prior is also calculated for achieving the expected outcome. My design achieves the following goals.

\subsubsection{Encoding flexibility}

Different mobile devices have uniquely designed displays, customized video quality playback service, hardware, and various codec types. Recent conventional solutions would adopt a few encoding formats ahead of the release of a video program. But even the most popular and magnanimous content providers would not be able to achieve and adapt to all possible mobile platform and hardware specification features. Cloud based Mobile Streaming customizes the streams for different devices at real time, by offloading the trans-coding tasks to an IaaS cloud. In particular, we novelty employ a surrogate for each user, which is a virtual machine (VM) in the IaaS cloud. The surrogate (foster machine) downloads the video on behalf of the user and trans-codes it into the destined formats, while catering to the specific configurations of the mobile device as well as the current network quality. 


\subsubsection{Battery efficiency}

A breakdown analysis conducted in earlier research indicates that the network modules (both $\mathrm{Wi}-\mathrm{Fi}$ and $3 \mathrm{G}$ ) and the display contributes to a significant portion of the overall power consumption in a mobile device, reducing usages from other hardware modules including CPU storage, memory, etc. Targets are usually over energy savings from the network module of smart-phones through an efficient data transmission mechanism design. Focus is made basically on $3 \mathrm{G}$ wireless networking as it is getting more widely used and challenging in our design than Wi-Fi based transmissions. Based on cellular network workings of real-world $3 \mathrm{G}$ carriers, verification are made in the $3 \mathrm{G}$ configuration parameters such as the power states and the inactivity timers, and design a novel burst transmission and spatial resolution control mechanism for streaming from the surrogate machines to the user end devices. This transmission mechanism makes careful decisions on opportunistic transitions among high/low power consumption modes at the devices, by effectively increasing the battery lifetime and display characteristics.

\subsubsection{Portability}

A prototype Cloud based Mobile Streaming is implemented following the philosophy of "Write Once, Run Anywhere" (WORA): both the front-end mobile modules and the backend server modules are implemented, with well-designed generic data models suitable for any Big Table-like data store; the only exception being the trans-coding module. The client module can run on any mobile devices supporting HTML5, including Android phones, iOS and all smart phone devices.

\section{CONCLUSION}

The Mobile multimedia streaming services aims at providing appropriate multimedia files according to the network and device features. A set of adaptive networks and a device and energy aware QoS approach for interactive mobile streaming is proposed. The ANCM and BPNDM were used for the prediction of network and device features, and the communication frequency and SVC multimedia streaming files most suitable for the device environment were determined according to these two modules. In the experiment, the overall prototype architecture needs to be realized and an experimental analysis ought to be carried out. The proposed prototype supports and maintains a certain level of multimedia service quality for dynamic network environments and always ensures smooth and complete multimedia streaming services. Media-Cloud services may accelerate research on SVC coding in the future.

\section{REFERENCES}

[1] W. Song, J. Hai, C. Jie, and F. Kaiqin, "A novel cache scheme for cluster-based streaming proxy server," in Proc. IEEE Int. Conf. Distributed Computing Syst. Workshops, 2005, pp. 727-733.

[2] Y. Hang, C. C. J. Kuo, and I. Ahmad, "Energy efficiency in data centers and cloud-based multimedia services: An overview and future directions," in Proc. Int. Green Computing Conf., 2010, pp. 375-382.

[3] P. Fan, J. Wang, Z. Zheng, and M. R. Lyu, "Toward optimal deployment of communication-intensive cloud applications," in Proc. IEEE Int. Conf. Cloud Computing, 2011, pp. 460-467.

[4] Z. Zheng, Y. Zhang, and M. R. Lyu, "Cloudrank: A QoSdriven component ranking framework for cloud computing," in Proc. IEEE Symp. Reliable Distributed Syst., 2010, p. 18493.

[5] D.Diaz-Sanchez,F.Almenarez,A.Marin,D. Proserpio, and P.A.Cabarcos, "Media cloud:An open cloud computing middleware for content management," IEEE Trans. Consum. Electron., vol. 57, pp.970-978, 2011.

[6] J. Feng, P. Wen, J. Liu, and H. Li, "Elastic stream cloud (ESC): A stream-oriented cloud computing platform for rich internet application,"in Proc. Int. Conf. High Performance Computing and Simulation (HPCS), 2010, pp. 203-208.

[7] I. Trajkovska, J. Salvachúa, and A. M. Velasco, "A Novel P2P and Cloud Computing Hybrid Architecture for Multimedia Streaming with QoS Cost Functions," Proc. ACM Multimedia, p. 1227230, 2010.

[8] Y.Liu,Z.Yang, X. Deng, J.Bu, andC.Chen, "Media browsing formobile devices based on resolution adaptive recommendation," in Proc.Int. Conf. Commun. and Mobile Computing, 2009, pp. 285-290.

[9] H. Sohn, H. Yoo, Y. B. Lee, C. S. Kim, W. D. Neve, and Y.M.Ro,"MPEG-21-Based scalable bitstream adaptation using medium grain scalability," in Proc. IEEE Region 10 Conf., 2008, pp. 1-5.

[10] H.Choi, J.W.Kang, and J. G. Kim, "Dynamic and interoperable adaptation of SVC for QoS-enabled streaming," IEEE Trans. Consum. Electron.,vol. 53, no. 2, pp. 384-385, 2007.

[11] H. Lee, Y. Lee, J. Lee, D. Lee, and H. Shin, "Design of a mobile video streaming system using adaptive spatial resolution control," IEEE Trans. Consum. Electron., vol. 55, no. 3, pp. 1682-1689, 2009. 